\title{
ON PERMUTATION POLYNOMIALS WHOSE DIFFERENCE IS LINEAR
}

\author{
by W. W. STOTHERS
}

(Received 7 November, 1988; revised 15 December, 1988)

1. Introduction. Let $q$ be a power of a prime $p$, and let $S_{q}$ be the set of permutations of $\{0,1, \ldots, q-1\}$. As $S_{q}$ is isomorphic to the group of permutations of $F_{q}$, the field of $q$ elements, each element of $S_{q}$ can be regarded as a polynomial over $F_{q}$. Various authors (e.g. [1], [2], [3]) have considered functions $f(x)$ such that

$$
f(x) \in S_{q}, \quad \text { and } \quad(f(x)+\lambda x) \in S_{q}
$$

for some $\lambda \in F_{q}$. When $\lambda=1, f(x)$ is a complete mapping polynomial ([3]).

Here, we consider the $f(x)$ for which there are several $\lambda$. For $q$ prime, such functions arose in (unpublished) work of $\mathbf{M}$. J. Tomkinson on group theory.

Definition. For $f(x) \in F_{p}[x]$,

and

$$
W_{f}=\left\{\lambda \in F_{q}:(f(x)+\lambda x) \in S_{q}\right),
$$

$$
w_{f}=\left|W_{f}\right| \text {. }
$$

Observe that, if $f(x) \in S_{q}$, then $0 \in W_{f}$, so that $w_{f} \geqslant 1$. Also, $f$ is a complete mapping polynomial if and only if $0,1 \in W_{f}$. On the other hand, if $(f(x)+\lambda x) \in S_{q}$, then we must have

i.e.

$$
f(0)+\lambda 0 \neq f(1)+\lambda 1
$$

$$
\lambda \neq f(0)-f(1)
$$

Thus $w_{f} \leqslant q-1$.

If we take $f(x)=\alpha x+\beta$, then

$$
f(x)+\lambda x=(\alpha+\lambda) x+\beta,
$$

so $\lambda \in W_{f}$ except when $\lambda=-\alpha$. We have proved

Proposition 1. If $f$ is a linear or constant polynomial over $F_{q}$, then $w_{f}=q-1$.

Tomkinson asked how large $w_{f}$ could be for non-linear $f$. We shall establish an upper bound, and discuss the $f$ which attain the bound.

Definition. A polynomial $f(x)$ over $F_{q}$ is reduced if the degree of $f$ is less than $q$.

Definition. If $f \in F_{q}[x]$, then we write $r_{q}(f)$ for the unique reduced polynomial equal to $f$ (as a function), and $d_{q}(f)$ for the degree of $r_{q}(f)$.

Proposition 2. If $f, g \in F_{q}[x]$ and $d_{q}(f)+d_{q}(g)<p$, then

and

$$
r_{q}(f g)=r_{q}(f) r_{q}(g)
$$

$$
d_{q}(f g)=d_{q}(f)+d_{q}(g)
$$

Glasgow Math. J. 32 (1990) 165-171. 
Proof. This follows at once from the uniqueness of the reduced polynomial.

We use (and then generalize) the following result from [1].

Lemma. A polynomial $f \in F_{q}[x]$ belongs to $S_{q}$ if and only if

and

(1) $f$ has a unique root in $F_{q}$,

$$
\text { (2) for } 1 \leqslant n \leqslant q-2, d_{q}\left(f^{n}\right) \leqslant q-2 \text {. }
$$

\section{The case $q$ prime.}

THEOREM 1. If $f \in S_{p}$ and $1 \leqslant m \leqslant w_{f}$, then

$$
d_{p}\left(f^{m}\right) \leqslant p-2+m-w_{f}
$$

Proof. From the Lemma, for $0<t<p-1$,

$$
r_{p}\left(f^{t}(x)\right)=\sum_{j=0}^{p-2} a_{t j} x^{j} .
$$

Then, for $0<t<p-1$, the coefficient of $x^{p-1}$ in $r_{p}\left((f(x)+\alpha x)^{t}\right)$ is

$$
\sum_{s=1}^{t-1}\left(\begin{array}{l}
t \\
s
\end{array}\right) \alpha^{s} a_{(t-s)(p-1-s)} .
$$

This is a polynomial in $\alpha$ of degree at most $t-1$, so, if non-zero, has at most $t-1$ roots in $F_{p}$. Thus, if $t \leqslant w_{f}$, it must be the zero polynomial, i.e. for $s \leqslant t \leqslant w_{f}$,

$$
a_{(t-s)(p-1-s)}=0 \text {. }
$$

Put $m=t-s$. Then, for $m \leqslant t \leqslant w_{f}$,

$$
a_{m(p-1+m-t)}=0 .
$$

Since this holds for $t \leqslant w_{f}$,

as required.

$$
d_{p}\left(f^{m}(x)\right) \leqslant p-2+m-w_{f}
$$

Remarks. (i) Dickson's result (our "Lemma") and Theorem 1 of [3] give the result for the cases $m=1,2$.

(ii) The proof fails for a prime to a power greater than one since some of the binomial coefficients in (2) vanish in $F_{q}$; see $\$ 3$.

In Proposition 1 , we saw that if $d_{p}(f) \leqslant 1$ then $w_{f}=p-1$. We now show that only linear $f$ have $w_{f}>(p-3) / 2$.

THEOREM 2. If $f \in S_{p}$ has $w_{f}>(p-3) / 2$, then $d_{p}(f) \leqslant 1$.

Proof. We may as well assume that $f$ is reduced. It is easily checked that all $f \in S_{2}$ are linear, so we may assume that $p>2$. Let $D$ be the degree of $f$. We suppose that $D>2$. By the Lemma applied to $f^{(p-1) / D}$, we see that $D \nmid(p-1)$, and hence that $p \geqslant 5$.

Let $m$ be the integer such that

$$
(p-1) /(m+1)<d<(p-1) / m,
$$


so $1 \leqslant m \leqslant(p-3) / 2$. Then $f^{m}$ is reduced and $d_{p}\left(f^{m}\right)=m D \leqslant(p-3) / 2+m$, by Theorem 1. It now follows from (3) that

$$
m(p-1) /(m+1)<(p-3) / 2+m .
$$

This implies that $(m-1)(p-3-2 m)<0$, a contradiction.

In particular, this shows that, for $p \leqslant 5$, the only polynomials $f$ with $w_{f}>1$ are linear. For $p>5$, we can have non-linear examples, as we shall see later.

Proposition 3. Suppose that $f \in F_{p}[x]$, and that $\alpha, \beta, \gamma \in F_{p}$, with $\alpha \neq 0$. Let $g \in F_{p}[x]$ be defined by $g(x)=\alpha f(x+\beta)+\gamma$. Then

and

$$
W_{g}=\left\{\alpha \lambda: \lambda \in W_{f}\right\}
$$

$$
w_{g}=w_{f}
$$

Proof. The second part follows at once from the first. To prove the first, we observe that $(g(x)+\mu x) \in S_{p}$ if and only if it is injective. Now

$$
g(x)+\mu x=g(y)+\mu y
$$

if and only if

$$
\alpha f(x+\beta)+\mu x=\alpha f(y+\beta)+\mu y .
$$

We choose $\lambda$ so that $\mu=\alpha \lambda$. Then the latter becomes (adding $\alpha \lambda \beta$ to each side)

$$
\alpha f(x+\beta)+\alpha \lambda(x+\beta)=\alpha f(y+\beta)+\alpha \lambda(y+\beta) .
$$

Hence $\mu \in W_{g} \Leftrightarrow \lambda \in W_{f}$.

Definition. For reduced $f, g \in F_{p}$ we write $f \rho g$ if there exist $\alpha, \beta, \gamma \in F_{p}$ with $\alpha \neq 0$ such that

$$
g(x)=\alpha f(x+\beta)+\gamma .
$$

Proposition 4. Each $\rho$-class of non-constant reduced polynomials in $F_{p}[x]$ contains a unique member of the form

$$
g(x)=x^{d}+\alpha_{d-2} x^{d-2}+\ldots+\alpha_{1} x .
$$

If $d=1$, then the class has $p(p-1)$ members; otherwise it has $p^{2}(p-1)$.

We leave the proof to the reader.

Definition. We say that a polynomial of the form (5) is normalized.

Theorem 3. For $p>5, f \in S_{p}$ has $w_{f}=(p-3) / 2$ if and only if $f$ is $\rho$-equivalent to

$$
g(x)=x^{(p+1) / 2}+a x
$$

for some $a \in F_{p}$.

Proof. We may as well assume $f$ is reduced. Let $D$ be the degree of $f$. By Theorem 1,

$$
D \leqslant(p-2)-(p-3) / 2+1=(p+1) / 2 \text {. }
$$


Suppose that $D<(p+1) / 2$. Arguing as in Theorem 2 (but with $w_{f}=(p-3) / 2$ ), we must have $p>7$ and, for some $m$ with $2 \leqslant m \leqslant(p-5) / 2$,

$$
(m-1)(p-3-2 m)<m+1 \text {. }
$$

Since $p-3-2 m \geqslant 2$, this gives a contradiction unless $m=2$. But then, as $p>7$, $p-3-2 m=p-7 \geqslant 4$, so we get a contradiction here also. Hence we must have $D=(p+1) / 2$.

Now let $g$ be the normalised polynomial $\rho$-equivalent to $f$. Then $g$ has degree $(p+1) / 2$ and

$$
g(x)=x^{(p+1) / 2}+\alpha x^{k}+\text { terms of lower degree, }
$$

where $k \leqslant(p-3) / 2$. We note that $\left(x^{(p+1) / 2}\right)^{2}$ reduces to $x^{2}$. Thus

$$
r_{p}\left(g^{2}(x)\right)=\left(2 \alpha x^{k+(p+1) / 2}+\ldots\right)+x^{2} .
$$

From Theorem 1, we have

$$
\begin{aligned}
d_{p}\left(g^{2}(x)\right) & \leqslant(p-2)-(p-3) / 2+2 \\
& =(p+3) / 2 .
\end{aligned}
$$

If $\alpha \neq 0$, then we must have $k+(p+1) / 2 \leqslant(p+3) / 2$, so that $k \leqslant 1$. As $g$ is normalised (so has no constant term),

$$
g(x)=x^{(p+1) / 2}+a x .
$$

To complete the proof, we must show that each $g$ of the form (5) has $w_{g}=(p-3) / 2$. We recall that $x^{(p-1) / 2} \equiv(x / p)$ (the Legendre symbol) (modulo $p$ ) so that

$$
g(x)+\lambda x=\left\{\begin{array}{cl}
0 & \text { if } x=0, \\
(a+\lambda+1) x & \text { if } x \text { is a quadratic residue modulo } p, \\
(a+\lambda-1) x & \text { otherwise. }
\end{array}\right.
$$

Since for all residues (resp. non-residues) $x,(a+\lambda+1) x$ (resp. $(a+\lambda-1) x)$ will have the same quadratic character, $(g(x)+\lambda x) \in S_{p}$ if and only if $(a+\lambda+1)$ and $(a+\lambda-1)$ have same quadratic character, i.e. for some $\alpha \neq 0$,

i.e.

$$
(a+\lambda+1)=\alpha^{2}(a+\lambda-1)
$$

$$
\lambda=\frac{\alpha^{2}+1}{\alpha^{2}-1}-a .
$$

Since there are $(p-3) / 2$ distinct squares modulo $p$, other than 0 and 1 , there are $(p-3) / 2$ valid $\lambda$, as required.

COROLlary 1. For $p>5$, there are $p^{3}(p-1)$ non-linear functions $f$ with $w_{f}=$ $(p-3) / 2$.

Proof. From the proof above, there are $p$ normalised functions $g$, and (by Proposition 4), each corresponds to $p^{2}(p-1)$ functions $f$. 
Remark. It is probably neater to re-cast the description of " $g$ " in Theorem 3 as

$$
g(x)= \begin{cases}0 & \text { if } x=0, \\ A x & \text { if } x \text { is a quadratic residue modulo } p, \\ B x & \text { otherwise, }\end{cases}
$$

where $A$ and $B$ are distinct modulo $p$, but of the same quadratic character. This form would have helped to simplify the discussion of [2].

Corollary 2 (c.f. Theorem 8 of [3]). For $p>5$ there exist non-linear complete polynomial mappings of $F_{p}$.

Proof. Since $p>3$, we can choose $A$ and $B$ distinct quadratic residues, and define $g$ by (6). As $(A / p)=(B / p)=1,0 \in W_{g}$. Now $w_{g}=(p-3) / 2 \geqslant 2$ (as $p>5$ ), so we have $\lambda \in W_{g}, \lambda \neq 0$. Now apply Proposition 3 to $\mu g$ (with $\mu \lambda \equiv 1$ (modulo $p$ ) to see that $0,1 \in W_{\lambda g}$, i.e. that $\lambda g$ is of the required type.

The ideas above can be used to construct other non-linear $f$ with $1<w_{f}<(p-3) / 2$ as follows.

Construction. If $p>5$, choose $h$ such that $h \mid(p-1)$ and $2<h<(p-1) / 2$. Let $\chi_{h}$ denote the $h$ th power residue symbol. Choose $A, B$ distinct members of $F_{p}$ and define $g(x)$ on $F_{p}$ by

$$
g(x)= \begin{cases}0 & \text { if } x=0 \\ A x & \text { if } \chi_{h}(x)=1 \\ B x & \text { otherwise }\end{cases}
$$

Much as before, $\lambda \in W_{g}$ if and only if

$$
(A+\lambda)=\alpha^{h}(B+\lambda)
$$

for some non-zero $\alpha \in F_{p}$. Rearranging:

$$
\lambda=\left(A-B \alpha^{h}\right) /\left(\alpha^{h}-1\right) .
$$

Since $\alpha^{h}$ takes $((p-1) / h)-1$ distinct values other than 1 , we have

$$
w_{g}=((p-1) / h)-1
$$

We observe that, as a polynomial,

$$
g(x)=x\left(A\left(x^{p-1}-1\right) /\left(x^{(p-1) / h}-1\right)+B\left(x^{h}-1\right)\right.
$$

so that

$$
\left.d_{p}(g)=1+(p-1)-(p-1) / h=p-2-((p-1) / h)-1\right)+1
$$

giving equality in Theorem 1.

We can, of course, modify the construction above to introduce $A$ 's for each residue class. This gives greater flexibility, and allows us to prove that we need not have equality in Theorem 1.

ExAMPLE. For $p=13$, let $f(x)=6 x\left(x^{4}+6 x^{2}+4\right)$. It is a simple matter to check that $w_{f}=\{0,1\}$, but $d_{p}(f)$ is only 5 . 
This example arises from 6th power residues (hence the appearance of even powers inside the brackets). There are, however, many $f$ with $1<w_{f}<(p-3) / 2$ which do not arise from our construction (these of necessity have $w_{f}=((p-1) / h)-1$ for some $h$; for example, there are 110 classes in $F_{11}[x]$ with $w_{f}=3$ ).

3. The general case. As noted earlier, the proof of Theorem 1 fails for $q=p^{r}$ with $r>1$. We can prove a weaker version which shows that, except in special cases, a reduced $f \in F_{q}[x]$ with $w_{f}>1$ begins with powers $x^{d}$, where $p \mid d$. The result is incomplete, so we omit it. Based on our experimental evidence (see section 4) we are, however, prepared to make the following conjecture.

CONJECTURE. If $f \in F_{q}[x]$ with $w_{f}>(p-3) / 2$, then $f(x)=g^{p}(x)+a x$, where $d_{p}(g) \leqslant q / p$.

We give an example to show that the situation is more complicated than that in $\$ 2$.

ExAmple. Let $f(x)=x^{q}-a x$. Then, calculating in $F_{q^{2}}, f(x)=f(y)$ if and only if

$$
a=\left(x^{q}-y^{q}\right) /(x-y)=(x-y)^{q} /(x-y) .
$$

Thus, $f(x)$ fails to permute $F_{q^{2}}$ if and only if $a$ is a $(q-1)$ th power, i.e. $a^{q+1}=1$. Since there are $q+1$ elements with this property,

$$
w_{f}=\left(q^{2}-1\right)-(q+1)+1=q^{2}-q-1
$$

REMARK. The construction introduced in $\$ 2$ works for prime powers, provided that the value of $h$ is prime to $p$. These seem to account for "large" values of $w_{f}$. We have proved this for $q=4,8,9,16$ and 25 , verifying the experimental results in the first four cases.

4. Experimental results. We have written a program which checks each permutation $f(x)$ to see whether $f(x)+x$ is also a permutation, and, if so, finds the $\lambda$ for which $f(x)+\lambda x$ belongs to $S_{q}$. For each such $f$, it checks the degree of the reduced version of $f$.

These computations take a considerable time (hours of mainframe time), so it is unlikely to be sensible to carry them much further.

We show below the total number, $C_{q}$, of complete mapping polynomials for small values of $q$. Of course, the actual output was much more detailed. The results of the calculations suggest that the permutations are not randomly spread amongst the polynomials. Since there are $q$ ! permutations and $q^{q-1}$ polynomials of degree at most $q-2$, a random distribution would predict about $(q !)^{2} / q^{q-1}$ polynomials $f(x)$ with $f(x)+x$ also in $S_{q}$.

\begin{tabular}{rrr}
$q$ & \multicolumn{1}{c}{$C_{q}$} & $(q !)^{2} / q^{q-1}$ \\
5 & 20 & 25 \\
7 & 133 & 216 \\
8 & 384 & 775 \\
9 & 2241 & 3059 \\
11 & 37851 & 61431 \\
13 & 1030367 & 1664334 \\
16 & 244744192 & 379698995 \\
17 & 1606008513 & 2599885897
\end{tabular}


The figures in the last column are rounded to the nearest integer. We observe that, for prime $q$, the ratio $C_{q} /(q !)^{2} q^{q-1}$ is remarkably close to the golden section!

Acknowledgement. I should like to thank the referee for suggesting a better way of presenting the argument in Theorems 2 and 3.

\section{REFERENCES}

1. L. E. Dickson, Linear Groups (Dover, 1958).

2. G. Mullen and $H$. Niederreiter, The structure of a group of permutation polynomials, $J$. Austral. Math. Soc. Ser. A 38 (1985), 164-170.

3. H. Niederreiter and K. H. Robinson, Complete mappings of finite fields, J. Austral. Math. Soc. Ser. $A 33$ (1982), 197-212.

Department of Mathematics, UNIVERSITY GARDENS, GLASGOW G12 8QW 